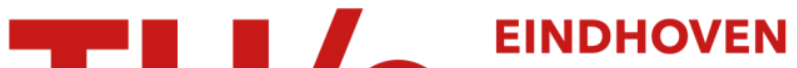 \\ UNIVERSITY OF \\ TECHNOLOGY
}

\section{Capacity assignment in repair shops with high material uncertainty}

\section{Citation for published version (APA):}

Driessen, M., van Houtum, G-J., Zijm, H., \& Rustenburg, W. D. (2020). Capacity assignment in repair shops with high material uncertainty. International Journal of Production Economics, 221(1), [107484].

https://doi.org/10.1016/j.ijpe.2019.09.005

DOI:

10.1016/j.jpe.2019.09.005

Document status and date:

Published: 05/03/2020

\section{Document Version:}

Accepted manuscript including changes made at the peer-review stage

\section{Please check the document version of this publication:}

- A submitted manuscript is the version of the article upon submission and before peer-review. There can be important differences between the submitted version and the official published version of record. People interested in the research are advised to contact the author for the final version of the publication, or visit the $\mathrm{DOI}$ to the publisher's website.

- The final author version and the galley proof are versions of the publication after peer review.

- The final published version features the final layout of the paper including the volume, issue and page numbers.

Link to publication

\section{General rights}

Copyright and moral rights for the publications made accessible in the public portal are retained by the authors and/or other copyright owners and it is a condition of accessing publications that users recognise and abide by the legal requirements associated with these rights.

- Users may download and print one copy of any publication from the public portal for the purpose of private study or research.

- You may not further distribute the material or use it for any profit-making activity or commercial gain

- You may freely distribute the URL identifying the publication in the public portal.

If the publication is distributed under the terms of Article 25fa of the Dutch Copyright Act, indicated by the "Taverne" license above, please follow below link for the End User Agreement:

www.tue.nl/taverne

Take down policy

If you believe that this document breaches copyright please contact us at:

openaccess@tue.nl

providing details and we will investigate your claim. 


\title{
Capacity assignment in repair shops with high material uncertainty
}

\author{
M.A. Driessen ${ }^{1,3, *}$, G.J. van Houtum ${ }^{1}$, W.H.M. Zijm² ${ }^{2}$ W.D. Rustenburg ${ }^{3}$ \\ ${ }^{1}$ Eindhoven University of Technology, School of Industrial Engineering \\ P.O. Box 513, 5600 MB Eindhoven, the Netherlands \\ ${ }^{2}$ University of Twente, Dept. Industrial Engineering and Business Information Systems \\ P.O. Box 217, 7500 AE Enschede, the Netherlands \\ ${ }^{3}$ Gordian Logistic Experts, \\ Kerkweg 10, 3603 CM Maarssen, the Netherlands
}

September 8, 2019

\begin{abstract}
We consider a group of identical systems, each consisting of multiple Line Replaceable Units (LRUs) that fail according to a Poisson process. A failed LRU is replaced by a ready-for-use one from a single stock point and, if not available, a backorder cost is incurred per unit of time. The failed LRU is returned to a repair shop, where it is inspected to identify which Shop Replaceable Units (SRUs) caused the failure, and is repaired by replacing the failed SRUs. After repair the LRU is ready-for-use again. Both the LRUs and SRUs are controlled by base stock policies. The repair shop is modeled as a two-stage service process consisting of an inspection and a repair phase. Inspection and repair are executed by one group of repairmen. The repair times depend on the time that elapses between inspection and the repair of a part. We model the total repair capacity as a single server and we compare policies that, based on the repair workload in the repair shop, give priority to either inspection or repair of parts. We suggest two approaches to set the SRU base stock levels, and simulate the system for multiple combinations of the repair workload threshold and predetermined vectors of SRU base stock levels. Based on the simulation results, the LRU base stock levels are optimized. We study a representative setting in which the repair shop faces a high material uncertainty, under different scenarios. We show that a scenario in which we maximize the $S R U$ job completeness, combined with a repair priority policy (in which repair of jobs takes precedence over inspection of jobs) leads to the lowest total costs.
\end{abstract}

Keywords: Repairable spare parts, Multi-item two-indenture system, Two-stage repair system, Priorities, Simulation

\section{Introduction}

In many industries, companies depend on the availability of high-value capital assets to provide their services or to manufacture their products. Examples are manifold, e.g. airlines, public and private transport organizations, hospitals, defense organizations, high-tech manufacturing companies and

\footnotetext{
${ }^{*}$ Corresponding author, E-mail: m.driessen@gordian.nl
} 
process industries. Periods in which the asset is not available for use may among others result in lost revenues, customer dissatisfaction and possible claims or a public safety risk. For optimal functioning and availability of the assets, timely maintenance of the assets is essential.

When maintenance of an asset is executed, parts may be taken out and replaced by ready-foruse parts based on their condition (the "repair-by-replacement" principle). These parts are called Line Replaceable Units (LRUs), and sometimes they are entire modules consisting of multiple parts. Modules that are taken out and appear to be irrepairable (or are not worth the effort) are discarded. In the other case, they are returned to a repair shop and disassembled and inspected to find the malfunctioning parts, which then are replaced by well-functioning ones after which the module is assembled and added to a ready-for-use inventory. The replaced parts, called Shop Replaceable Units (SRUs), may be processed similarly. In general, an asset may consist of several indenture levels (module, part, part-component, etc.).

Systems in which a repair shop returns (failed) repairable parts to ready-for-use (RFU) stock are denoted as repairable parts inventory systems (Driessen, 2018). Repair shops may have different levels of capacity complexity and material uncertainty. Driessen et al. (2015) present a typology of repair shops which is based on shops having similar characteristics (see Figure 1). In this paper we restrict ourselves to a repair shop of type II, i.e. a repair shop with a low capacity complexity and a high material uncertainty. A high material uncertainty means that the sets of SRUs that are required to repair parts of the same LRU strongly vary, and that a large set of SRUs has to be kept on stock; a low capacity complexity means that the repair capacity is homogeneous and the number of specialized skills is low (Driessen et al., 2015).

Decision makers in repairable parts inventory systems have to take into account trade-offs between SRU and LRU base stock levels, repair capacities, repair shop scheduling rules and the rules for allocation of SRUs to inspected failed parts. In models that analyze the design of such systems, repair capacity is either not modeled explicitly (the "METRIC"-type models, see e.g. Sherbrooke (1986)) or it is assumed that failed repairable parts are inspected upon failure, in a negligible time (e.g. Sleptchenko et al. (2003)). Furthermore, it is often assumed that at most one SRU causes the failure of a next higher indenture level part.

In this paper we introduce three new aspects that are currently not covered in the literature, but that have been observed in practice (see Chapters 3 and 5 in Driessen (2018)). First, we model 


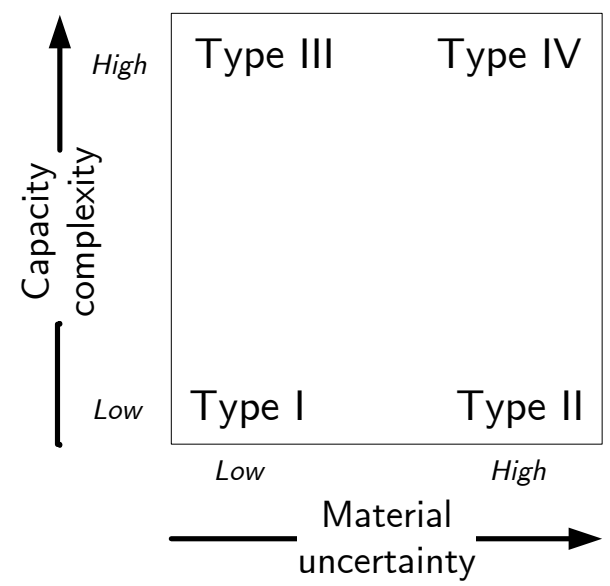

Figure 1: Typology of repair shops for repairable parts.

the repair shop as a two-stage service process consisting of an inspection and a repair phase, that both may take a considerable amount of time and are executed by the same resources. Each failed part follows this two-stage service process and constitutes a so-called service job. Second, repair times depend on the time that elapses between inspection and the repair of a part. Repair times are higher for interrupted service jobs, because the inspected part needs to be put aside and the repair man has to go into the problem again, i.e. a part of the inspection has to be redone when the repair starts. Third, we take into account that multiple SRUs may be required for the repair of a part, and the set of SRUs that is actually required for service jobs of the same LRU can vary from one service job to another.

These new aspects raise the question whether priority should be given to inspection of failed parts (to reveal which SRUs are required and speed up sourcing of unavailable SRUs) or to completion of the repair of parts for which all required SRUs are available (to increase the availability of LRUs). We model the total repair capacity as a single server and we compare (the total costs for) several capacity priority policies. We construct two 'extreme' policies (an inspection and a repair priority policy) in which inspection (repair) has priority, and multiple hybrid priority policies that, based on whether the repair workload in the shop is below or above a certain threshold, give priority to either inspection or repair of parts. The total costs include inventory holding costs and backordering costs, which clearly depend on the base stock levels of the SRUs and LRUs.

One would ideally compare the capacity priority policies under the optimal SRU and LRU 
base stock levels. However, these optimal base stock levels also depend on the capacity priority policy itself. To the best of our knowledge, models to analytically solve this problem are not known in the literature. Therefore we suggest two approaches to set the SRU base stock levels. In the first approach, all SRU base stock levels are set according to identical fill rate targets. In the second approach we maximize the "SRU job completeness", i.e. the probability that all SRUs for a random service job are directly available from stock, under an inventory holding costs constraint. We simulate the system for multiple combinations of the repair workload threshold and predetermined vectors of SRU base stock levels. Based on the simulation results, the LRU base stock levels are optimized. This leads to a fair comparison of capacity priority policies.

We construct a representative setting in which the repair shop faces a high material uncertainty, i.e. with multiple LRUs all consisting of relatively expensive SRUs having low demand rates, based on figures and observations from practice. From the scenarios we have evaluated, the scenario in which we maximize the SRU job completeness and high investments in SRUs, combined with a repair priority policy leads to the lowest total costs. In general, under a relatively high aggregate SRU fill rate, i.e. the weighted average fill rate over all SRUs, we observe that the repair priority policy performs best. In a setting with low to moderately high SRU fill rates, inspection and hybrid priority policies perform best.

The main contribution of this paper is the development of a new, realistic model that covers the three aspects mentioned above. We explicitly distinguish the inspection and repair phase of the service jobs and focus on the question whether inspection or repair should have priority, in combination with setting appropriate SRU stock levels. To the best of our knowledge, we are the first to make this distinction. This distinction, together with the feature that a failed LRU may need multiple SRUs for its repair, lead to a complex model for which it is hard to come to analytical results. Hence, much of our analysis will be based on simulations. We will choose the class of capacity priority policies such that we can optimize the LRU base stock levels, facilitating the analysis of large-scale problem instances as computation times are linear in the number of LRUs and SRUs.

This paper is organized as follows. In Section 2 we discuss related literature and in Section 3 we formulate our model. Section 4 describes the approaches we used to set the SRU base stock levels and Section 5 discusses the capacity priority policies that we evaluate. In Section 6 we describe 
how we set up the simulation experiment and in Section 7 we present a numerical study. Section 8 concludes this paper and lists some suggestions for further research.

\section{Literature review}

In repairable parts inventory systems, demand for a repairable part coincides with the return of a failed part that needs to be maintained, and a service job can only start after a demand occurred. This is similar to pull control systems, in which production (repair) is triggered by actual demands. In this review, we therefore concentrate on three literature (streams) related to pull control systems. Two well-known pull control systems are the base stock inventory policy and the Kanban control system (Buzacott and Shanthikumar (1993), Hopp and Spearman (2001)) without batching, i.e. in both systems we apply a one-for-one replenishment policy in which the use of an item to satisfy demand is immediately followed by an action to replenish the open stock position.

The first stream of literature is related to uncapacitated inventory models for determining inventory (base stock) policy parameters, and studies so-called "METRIC-type" models. Sherbrooke (1968) presents a multi-echelon, multi-item inventory model (METRIC), that is extended to a multi-indenture setting (MOD-METRIC) by Muckstadt (1973) and later on improved by Sherbrooke (1986), by means of the VARI-METRIC model, and by Rustenburg et al. (2003). We refer the reader to Sherbrooke (2004) and Van Houtum and Kranenburg (2015) for an overview. More recent papers in this stream study repair expediting in fluctuating demand environments (e.g. Levner et al. (2011), Arts (2017)) and shipment consolidation (e.g. Stenius et al. (2018)).

The "METRIC-type" models can be used to determine the optimal base stock levels of all LRUs and SRUs, given a limited budget for inventory investments, such that the average operational availability of the assets is maximized (i.e. the expected number of LRU backorders is minimized). To facilitate the analysis, these models require two important assumptions that may not always be justified. First, failed parts are repaired by replacing at most one part at a lower indenture level, whereas Van Jaarsveld et al. (2015) state that LRUs in general consist of multiple SRUs, and more than one may be required simultaneously for the repair of an LRU. Second, the repair lead times in these models are assumed to be independent and identically distributed (i.i.d.) random variables, and can have a general distribution of which only its mean value should be known. If the repair 
shop utilization is high, then this assumption leads to significant underestimation of stock levels and service performance (Sleptchenko et al. (2002)). The main difference between our work and the work in this stream is that we do not assume i.i.d. repair lead times and that multiple SRUs may be required simultaneously to repair a next higher indenture level part.

In case repair capacity and flexibility options are restricted, and resources are highly utilized, integrating capacity and inventory decisions may be worthwhile. The repair process may consist of one or multiple stages, and the complexity to solve capacitated inventory problems increases in the complexity of the scheduling rule that is used. In a single-stage setting, scheduling rules reduce to priority rules only, and hence priority and capacity allocation decisions coincide.

The second stream of literature is related to single-stage, capacitated inventory models, in which the repair shop has no capacity flexibility options. First papers in this stream of literature focused on deriving approximation methods to solve queuing type models with an exponential server for a single, multi-indentured LRU in a multi-echelon setting (e.g. Gupta and Albright (1992) and Albright and Gupta (1993)). We hereafter describe some extensions of these models to settings with multiple, multi-indentured LRUs and other priority rules.

Sleptchenko et al. (2003) model a trade-off between repair capacity and inventory, in which the repair shop is modeled as a multi-class $M / M / k$-queue. The number of servers is a decision variable and service jobs are executed on a "first-come, first-served" (FCFS) basis. Inspection of failed parts takes no time and is performed upon failure (which implies that repair activities may be preempted by inspection and diagnostics activities, but at no loss of repair time). Extending this model to an $M / G / c$-queue requires the assumption of at most one LRU.

Adan et al. (2009) have developed an exact model for simultaneously determining initial stocks and static repair priorities. They consider a multi-item, single-indenture model in which capacity is modeled as an $M / M / 1$-queue with multiple priority classes. Each LRU is assigned a priori to each of the priority classes (static priority rules), and SRUs are not included in the model, or are assumed to cause no delay of the repairs. Also Sleptchenko et al. (2005) developed a model comparable to that of Adan et al. (2009) which includes SRUs, though this model is applicable only for a small set of LRUs with at most two priority classes.

Static priority policies have the advantage that steady-state repair pipeline distributions can be determined (or approximated) independent of LRU base stock levels and actual on-hand stock 
levels. Relaxing this assumption makes an exact evaluation hard if multiple LRUs are considered. Chapter 3 of Tiemessen (2014) is an extension of Tiemessen and van Houtum (2013), and uses simulation optimization to determine near-optimal base stock levels for myopic policies in a single server, multi-item, single-indenture setting.

The main difference between our work and the work in the second stream is that we consider a setting with multiple LRUs having general service times. Multiple SRUs may be required to repair a failed LRU, and the SRU requirements are known only after the inspection phase, and inspection and repair both take a significant amount of time and are executed by the same resources.

The third stream of literature is related to multi-stage, capacitated inventory models. The Kanban control system is a way to model a manufacturing process consisting of multiple stages (Hopp and Spearman (2001)). The advantages of both the base stock policy (direct response) and the Kanban control system (i.e. resource constraints and a workload control rule) are combined in Generalized Kanban Control Systems (Buzacott (1989)) and Extended Kanban Control Systems (Frein et al. (1995) and Dallery and Liberopoulos (2000)). These systems integrate decisions regarding base stock levels and the number of Kanbans at each of the stages, and require the assumptions that stages have dedicated resources and that stages are visited in the same sequence. These stages can be regarded in multiple ways when looking at repairable parts inventory systems.

First, as has been noted by Zijm and Avşar (2003), the stages in the Generalized Kanban Control Systems can be regarded as the indenture levels of repairable parts. They present an approximation method to determine the base stock levels for a single-location, single LRU, twoindenture level model with two SRUs. The final stage can be referred to as the repair and assembly process of an LRU, and each preceding phase can be seen as the repair and assembly process of a lower indenture level SRU. Second, the stages can be regarded as the specific resources (e.g. different skill levels of the repair men) that are required in the repair process. Third, the stages can be regarded as the phases (disassembly and inspection, repair and assembly and final test) in the repair process. The applicability of these models is limited: the main difference with our work is that we consider a setting with multiple, multi-indentured LRUs and a two-stage repair system in which both stages share one resource type. 


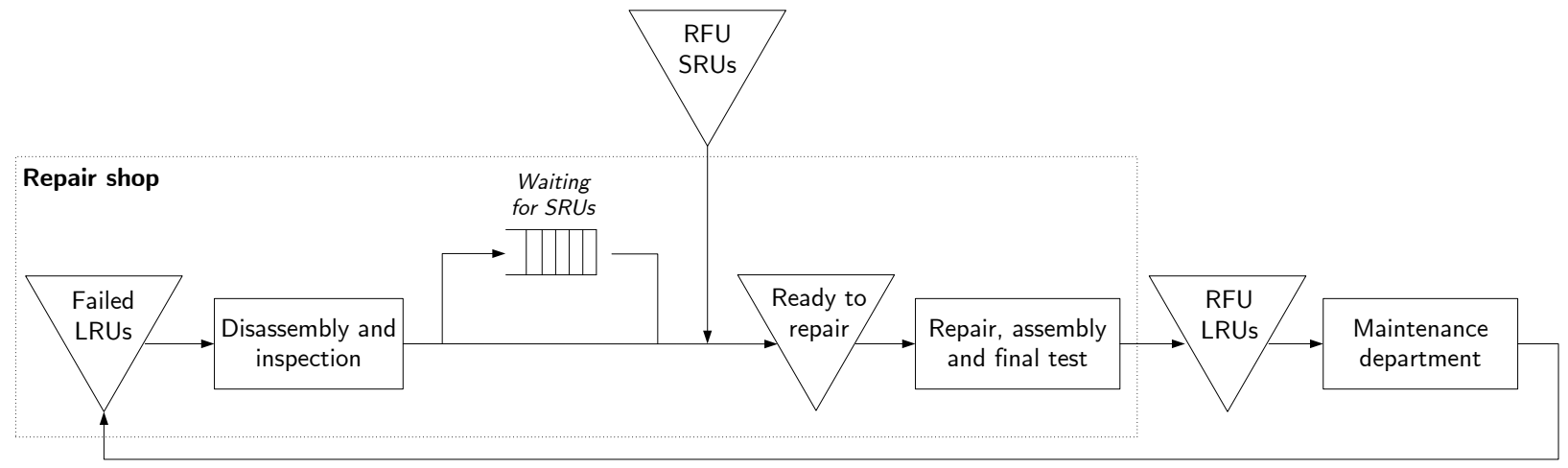

Figure 2: Overview of the general repair process

\section{Model description}

We consider a single-location, multi-item, two-indenture inventory model for repairable LRUs and a repair shop responsible for the repair of a fixed and finite set of repairable LRUs, denoted by $I$. The LRUs are numbered as $1, \ldots,|I|$. LRUs are used during first level maintenance of an asset, i.e. failed LRUs are replaced by ready-for-use (RFU) LRUs supplied from stock. The inventory of all LRUs is controlled by base stock policies and the vector of base stock levels is given by $\mathbf{S}=\left(S_{1}, \ldots, S_{|I|}\right)$. The inventory holding costs are denoted by $h_{i}>0$ for LRU $i$. Demands for LRU $i$ occur according to a Poisson process with constant rate $\lambda_{i}(\geq 0)$ and the demands are served FCFS. Unfulfilled demand for LRUs is backordered, and a backorder cost $b$ (independent of $i, b>0$ ) is incurred per part per time unit. We emphasize that in many situations high availability targets for LRUs are set, which correspond to cases where the backorder costs are much higher than the holding costs (i.e. $\left.b>>h_{i}, \forall i \in I\right)$.

The failed LRUs are sent to the stock point "Failed LRUs" and the return time is assumed to be equal to 0 (see Figure 2). Failed parts are inspected FCFS by the repair shop. The inspection phase of a service job cannot be interrupted (non-preemption) and the average inspection time equals $T_{i}^{\text {Insp }}$ time units for LRU $i$ (later we explain what is assumed for the underlying distribution of the inspection time).

Each service job may require a different set of SRUs. After the inspection phase, the set of required SRUs is known and demanded. Let $J=\{1, \ldots,|J|\}$ be the non-empty set of all SRUs. With probability $\alpha_{i, j}$ one unit of $\operatorname{SRU} j$ is needed for the repair of LRU $i$. Consequently, the 
aggregate demand rate $\left(\hat{\lambda}_{j}\right)$ for $\operatorname{SRU} j$ equals:

$$
\hat{\lambda}_{j}=\sum_{i \in I} \lambda_{i} \cdot \alpha_{i, j}, \quad \forall j \in J
$$

The inventory of all SRUs is controlled by base stock policies and the vector of base stock levels is given by $\hat{\mathbf{S}}=\left(\hat{S}_{1}, \ldots, \hat{S}_{|J|}\right)$. The inventory holding costs are denoted by $\hat{h}_{j}$ for SRU $j$. We assume that all SRUs are sourced at external suppliers (or repair shops) and a replenishment order for SRU $j$ is placed directly when a new part of SRU $j$ is demanded (directly after the inspection of a service job). SRU $j$ has a deterministic replenishment lead time $\hat{t}_{j}>0$.

Demands for SRUs are served FCFS and on-hand stock of SRUs, if available, is assigned to the service job that requires the SRUs. We distinguish assigned and unassigned on-hand SRU stock. Assigned on-hand SRU stock is stock that is already assigned to service jobs, that are waiting for other SRUs or waiting for repair. Unassigned on-hand stock of SRU $j$ is stock that is available to be assigned to new service jobs requiring $\operatorname{SRU} j$.

Let $M \subseteq J$ be the set of required SRUs for a random service job, let $M_{a} \subseteq M$ be the set of SRUs for which still unassigned on-hand stock is available and let $M_{u} \subseteq M$ be the set of SRUs for which no unassigned on-hand stock is available. After the inspection phase, for each SRU $j \in M_{a}$ the assigned on-hand stock is increased with one unit and the unassigned on-hand stock is decreased with one unit, but at the same time a replenishment order for one unit is placed. For each SRU $j \in M_{u}$, the number of backorders is increased with one unit (again, while placing a replenishment order). Once a replenishment order for SRU $j$ arrives, either the unassigned stock of SRU $j$ is increased or the number of backorders of SRU $j$ is decreased, and in the latter case also the assigned on-hand stock of SRU $j$ is increased.

Service jobs for which all required SRUs are available, are sent to the stock point "Ready to repair". All other service jobs are queued "waiting for SRUs" until the last required SRU has been assigned. Note that service jobs can pass each other when at least one of them is waiting for SRUs. The required SRUs are taken from the on-hand stock when the repair phase of the service job starts.

The repair phase of a service job cannot be interrupted (non-preemption) and takes on average $T_{i}^{R e p}$ time units for LRU $i$. If the repair phase does not start within $T^{M a x}(\geq 0)$ time units after 
completion of the inspection phase, a factor $\delta_{i}$ multiplied by the service job inspection time is required in addition to the repair time, with $0 \leq \delta_{i} \leq 1$. This additional time is referred to as 'inefficiency', and $\delta_{i}$ is referred to as the 'inefficiency factor' for LRU $i$ (note: it is important to emphasize that this extra time is not a classical inspection again, since all parts needed are already available at this stage. It is only needed to fresh up the failure knowledge). After the repair phase, the part is "ready-for-use" again and sent to the ready-for-use LRU stock ("RFU LRUs").

We assume that the repair shop consists of a single server (repair man) that works with speed 1. The workload, i.e. the sum of inspection and repair time, of an efficiently executed service job for LRU $i$ has a general distribution with average $\mu_{i}$ and standard deviation $\sigma_{i}$ time units. The ratio between inspection and repair is constant for each (efficiently executed) service job: a fixed percentage of the workload of each (simulated) service job is required for inspection, denoted by $p_{i}$ for LRU $i$. Hence, $T_{i}^{I n s p}$ equals $p_{i} \mu_{i}$ and $T_{i}^{R e p}$ equals $\left(1-p_{i}\right) \mu_{i}$.

If not idle, the server works either on inspection or repair of a single service job. The selected capacity priority policy, denoted by $\pi$, determines when priority is given to either inspection or repair of parts. The server utilization $\rho$ depends on the SRU base stock levels and the capacity priority policy $\pi$ that is applied. The maximum utilization $\rho^{M a x}$ is based on the situation in which all service jobs are executed 'inefficiently', where $\rho \leq \rho^{M a x}$. The following condition is sufficient to ensure our system is stable:

$$
\rho^{M a x}=\sum_{i \in I} \lambda_{i} \cdot\left(\left(1+\delta_{i}\right) T_{i}^{I n s p}+T_{i}^{R e p}\right)<1
$$

We assume that $\pi \in \Pi$, where $\Pi$ is a class of capacity priority policies that dynamically assign capacity to either inspection or repair of parts, based on information in the repair shop. These capacity priority policies do not take into account information on the ready-for-use LRU stock, and hence the repair pipeline process of LRUs is independent of the base stock levels of the LRUs. This property is also referred to as: "the repair pipeline process is translation invariant" (see e.g. Kohler-Gudum and de Kok (2002)). With this property we are able to determine the optimal LRU base stock levels $\mathbf{S}^{*}(\hat{\mathbf{S}}, \pi)$, after we obtain the simulation results for a given vector $\hat{\mathbf{S}}$ and capacity priority policy $\pi$. 
Let $A_{i}(\hat{\mathbf{S}}, \pi, q)$ be the (steady state) probability that the number of parts of LRU $i$ in the repair shop equals $q$ (see Figure 2), $q \in \mathbb{N}_{0}$, given a vector of SRU base stock levels $\hat{\mathbf{S}}$ and capacity priority policy $\pi$. Further, let $B_{j}(\hat{\mathbf{S}}, \pi, k)$ be the (steady state) probability that the (assigned plus unassigned) on-hand stock of SRU $j$ equals $k, k \in \mathbb{N}_{0}$. The steady state probabilities of $A_{i}(\hat{\mathbf{S}}, \pi, q)$ and $B_{j}(\hat{\mathbf{S}}, \pi, k)$ will be determined with discrete event simulation, and both $\hat{\mathbf{S}}$ and $\pi$ are input variables for the simulation.

The average total system costs, given a vector of SRU base stock levels $\hat{\mathbf{S}}$, capacity priority policy $\pi$ and the vector of base stock levels for the LRUs (S), are defined as:

$$
C(\hat{\mathbf{S}}, \pi, \mathbf{S})=\sum_{j \in J} \sum_{k=0}^{\infty} \hat{h}_{j} \cdot k \cdot B_{j}(\hat{\mathbf{S}}, \pi, k)+\sum_{i \in I} h_{i} \cdot S_{i}+\sum_{i \in I} \sum_{q=S_{i}+1}^{\infty} A_{i}(\hat{\mathbf{S}}, \pi, q) \cdot\left(q-S_{i}\right) \cdot b
$$

The inventory holding costs for the SRUs (first part of the cost function) follow directly from the simulation results of the on-hand stock distribution $B_{j}(\hat{\mathbf{S}}, \pi, k)$. With the repair pipeline distribution $A_{i}(\hat{\mathbf{S}}, \pi, q)$, we are able to determine the optimal LRU spares vector $\mathbf{S}^{*}$. The total LRU costs are separable for each LRU $i$ and are equal to:

$$
C_{i}\left(\hat{\mathbf{S}}, \pi, S_{i}\right)=h_{i} \cdot S_{i}+\sum_{q=S_{i}+1}^{\infty} A_{i}(\hat{\mathbf{S}}, \pi, q) \cdot\left(q-S_{i}\right) \cdot b
$$

It is easy to see that an optimal base stock level of LRU $i$ is the smallest value $S_{i}^{*}$ that satisfies:

$$
\sum_{q=0}^{S_{i}^{*}} A_{i}(\hat{\mathbf{S}}, \pi, q) \geq \frac{b-h_{i}}{b}
$$

With the optimal base stock levels of the LRUs, we are able to determine the total system costs $C_{i}\left(\hat{\mathbf{S}}, \pi, S_{i}^{*}\right)$. As mentioned earlier, the vector of SRU base stock levels $\hat{\mathbf{S}}$ and the capacity priority policy $\pi$ are input variables for the simulation. How they are chosen is explained in Sections 4 and 5 , respectively. 


\section{Base stock levels of SRUs}

The SRU base stock levels are input variables for the simulation and hence need to be determined upfront. To do so, we need to make an assumption regarding the demand process of the SRUs. While LRU demands occur according to a Poisson process, SRU demands clearly do not. This is due to the fact that capacity (inspection) is required to determine the demands for SRUs, and hence demands for SRUs occur a certain (non-deterministic) time after the LRU demand occurred. In the remainder of this section, in which we describe two approaches to set the SRU base stock levels, we nevertheless assume that the SRU demands do occur according to a Poisson process. The first approach is rather simple, but we would like to mention that the derivation and description of the second approach is long.

\section{Approach 1: All SRUs have an identical fill rate target $\gamma$}

In this approach, the base stock level $\hat{S}_{j}$ of SRU $j$ is the smallest value for which the item fill rate of SRU $j$, denoted by $\beta_{j}\left(\hat{S}_{j}\right)$, is larger than or equal to $\gamma$, i.e.

$$
\beta_{j}\left(\hat{S}_{j}\right)=\sum_{x=0}^{\hat{S}_{j}-1} P\left(X_{j}=x\right) \geq \gamma, \quad \forall j \in J,
$$

where $X_{j}$ represents the number of parts in the replenishment pipeline of SRU $j$, which is assumed to be Poisson distributed with mean $\hat{\lambda}_{j} \hat{t}_{j}$.

\section{Approach 2: Maximize SRU job completeness, given a constraint on the holding costs for unassigned on-hand SRU stock}

In this approach, our objective is to maximize the probability that all SRUs for a random service job are available, given a target value for the holding costs. In the inventory model that we use, it is assumed that demand is served directly from stock, if available, and hence this model focuses on unassigned stock only. Therefore, we set a target value for the unassigned on-hand SRU stock

$C^{\text {obj }}$, despite the fact that the cost function in section 3 includes both assigned and unassigned on-hand SRU stock.

The probability that all SRUs for a random service job are available immediately after inspection 
will be referred to as 'SRU job completeness'. The SRU job completeness for LRU $i$, given a vector of SRU base stock levels $\hat{\mathbf{S}}$, is denoted by $\beta_{i}(\hat{\mathbf{S}})$. Let us now define the aggregate SRU job completeness as:

$$
\beta(\hat{\mathbf{S}})=\sum_{i \in I} \frac{\lambda_{i}}{\lambda} \beta_{i}(\hat{\mathbf{S}})
$$

where $\lambda=\sum_{i \in I} \lambda_{i}$. Notice that the SRU job completeness for a given individual service job of LRU $i$ is 1 in case each individual $\operatorname{SRU} j, j \in J$, is either (a) not required or (b) required and directly available from stock. In all other cases, the SRU job completeness is 0. Note that the SRU job completeness measure is equivalent to the immediate order fill rate for assemble-to-order systems. For such systems, it is well-known that due to correlation of demand across the components, the net inventories of the components are correlated random variables.

This applies to our problem in the following way. The demand processes of SRUs, that might simultaneously be required for the same service job, are correlated via the demand process of the parent LRU that requires these SRUs for repair. Hence, the steady-state distributions of the onhand stock of SRUs are correlated random variables. When demand processes of SRUs are strongly correlated, one might want to integrally determine the base stock levels of these SRUs.

Recall that $\alpha_{i, j}$ is the probability that SRU $j$ is required for the repair of LRU $i$ and $\beta_{j}\left(\hat{S}_{j}\right)$ is the fill rate of $\operatorname{SRU} j$. The probability that $\operatorname{SRU} j, j \in J$, is not required for a random service job of LRU $i$, is equal to $\left(1-\alpha_{i, j}\right)$. The probability that $\operatorname{SRU} j, j \in J$, is required for a random service job of LRU $i$ and available from stock, is equal to $\alpha_{i, j} \beta_{j}\left(\hat{S}_{j}\right)$. For low values of $\alpha_{i, j}$ and a large number of SRUs that might be required for the repair of an LRU, the correlation of the steadystate distributions of the on-hand stock of SRUs will be relatively low. Ignoring the correlations will not strongly influence the choice of the SRU base stock levels and we therefore assume that the steady-state distributions of the on-hand SRU stocks are uncorrelated. We can now write $\beta_{i}(\hat{\mathbf{S}})$ as:

$$
\beta_{i}(\hat{\mathbf{S}})=\prod_{j \in J}\left(\left(1-\alpha_{i, j}\right)+\alpha_{i, j} \beta_{j}\left(\hat{S}_{j}\right)\right)
$$


We can rewrite the SRU job completeness $\beta(\hat{\mathbf{S}})$ as:

$$
\begin{aligned}
\beta(\hat{\mathbf{S}}) & =\sum_{i \in I} \frac{\lambda_{i}}{\lambda} \prod_{j \in J}\left(1-\alpha_{i, j}\left(1-\beta_{j}\left(\hat{S}_{j}\right)\right)\right) \\
& \approx \sum_{i \in I} \frac{\lambda_{i}}{\lambda}\left(1-\sum_{j \in J} \alpha_{i, j}\left(1-\beta_{j}\left(\hat{S}_{j}\right)\right)\right) \\
& =1-\sum_{j \in J} \frac{\hat{\lambda}_{j}}{\lambda}\left(1-\beta_{j}\left(\hat{S}_{j}\right)\right) \\
& =1-\frac{1}{\lambda} \sum_{j \in J} \varphi_{j}\left(\hat{S}_{j}\right)
\end{aligned}
$$

where $\varphi_{j}\left(\hat{S}_{j}\right)=\hat{\lambda}_{j}\left(1-\beta_{j}\left(\hat{S}_{j}\right)\right)$ and where $\sum_{j \in J} \frac{\hat{\lambda}_{j}}{\lambda}\left(1-\beta_{j}\left(\hat{S}_{j}\right)\right)$ can be regarded as the 'weighted non-fill rate'. We would like to emphasize that the approximation used only holds for small values of $\alpha_{i, j}\left(1-\beta_{j}\left(\hat{S}_{j}\right)\right), \forall i \in I$. That is, for each combination of LRU $i \in I$ and SRU $j \in J$, either the fill rate $\beta_{j}\left(\hat{S}_{j}\right)$ is high, and/or the probability that SRU $j$ is required for the repair of LRU $i$ is small.

Let $C(\hat{\mathbf{S}})$ denote the total SRU holding costs (calculated over the unassigned on-hand SRU stock) and let $C_{j}\left(\hat{S}_{j}\right)$ denote the holding costs for SRU $j, \forall j \in J$, with:

$$
\begin{gathered}
C_{j}\left(\hat{S}_{j}\right)=\hat{h}_{j} \sum_{x=0}^{\hat{S}_{j}-1}\left(\hat{S}_{j}-x\right) P\left(X_{j}=x\right) \\
C(\hat{\mathbf{S}})=\sum_{j \in J} C_{j}\left(\hat{S}_{j}\right)
\end{gathered}
$$

We can now write our objective as the following problem $(P)$ : 


$$
\begin{gathered}
(P) \quad \max \quad \beta(\hat{\mathbf{S}}) \\
\text { subject to } C(\hat{\mathbf{S}}) \leq C^{o b j}, \\
\hat{\mathbf{S}} \in \mathbf{\Phi} .
\end{gathered}
$$

Solving problem $(P)$ is closely related to solving the following multi-objective programming problem $(Q)$ :

$$
\begin{array}{ll}
(Q) \quad \min & \sum_{j \in J} \varphi_{j}\left(\hat{S}_{j}\right) \\
\min & \sum_{j \in J} C_{j}\left(\hat{S}_{j}\right) \\
\text { subject to } \hat{S}_{j} \in \Phi_{j} \quad \forall j \in J .
\end{array}
$$

where $\Phi_{j}=\mathbb{N}_{0}$ represents the solution space for $\hat{S}_{j}, \forall j \in J$. For problem $(Q)$, we can derive socalled efficient solutions. In short, a solution is efficient if there exists no other solution for which the SRU job completeness is higher with the same SRU holding costs, or at least the same SRU job completeness can be attained with lower SRU holding costs. The set of efficient solutions form an efficient frontier for the total SRU holding costs and the SRU job completeness (or better: the 'non-fill rates' of SRUs). We can select an appropriate solution for problem $(P)$ from this efficient frontier.

In problem $(Q)$, the objective functions are separable for each SRU $j$ and therefore problem $(Q)$ as a whole is separable. It is easy to see that $\varphi_{j}\left(\hat{S}_{j}\right)$ is decreasing and convex on $\Phi_{j}^{\prime}=\left\{\hat{S}_{j} \in\right.$ $\left.\mathbb{N}_{0}: \hat{S}_{j} \geq \hat{\lambda}_{j} \hat{t}_{j}-1\right\}, \forall j \in J$ and $C_{j}\left(\hat{S}_{j}\right)$ is increasing and convex on $\mathbb{N}_{0}$. We now exclude solutions with $\hat{S}_{j}<\max \left\{\left\lceil\hat{\lambda}_{j} \hat{t}_{j}-1\right\rceil, 0\right\}$ from our solution space and discuss the excluded part.

The amount $\hat{\lambda}_{j} \hat{t}_{j}$ represents the average number of parts in the replenishment pipeline of SRU $j$. If the average replenishment pipeline stock is smaller than 1 for $\operatorname{SRU} j$, then $\Phi_{j}=\Phi_{j}^{\prime}$ and the solution space remains the same. If the replenishment pipeline stock is larger than 1 (which is in general not the case for slow moving parts), then we exclude solutions with $\hat{S}_{j}<\left\lceil\hat{\lambda}_{j} \hat{t}_{j}-1\right\rceil$. In 
problem instances with a high target for the SRU job completeness (i.e. a low value of $\sum_{j \in J} \varphi_{j}\left(\hat{S}_{j}\right)$ ), these solutions are generally not relevant. Besides this, the average number of parts on stock is low for all values of $\hat{S}_{j}<\left\lceil\hat{\lambda}_{j} \hat{t}_{j}-1\right\rceil$. Consequently, $C_{j}\left(\hat{S}_{j}\right)$ is also low in these cases and hence we would expect that, in an optimal solution, often the base stock levels of each $\operatorname{SRU} j \hat{S}_{j}$ will be set larger than or equal to $\left\lceil\hat{\lambda}_{j} \hat{t}_{j}-1\right\rceil$. For the latter reason, we would expect that reducing the solution space does not significantly influence the optimal solution even if the target for the SRU job completeness is low.

With the adjusted solution space, problem $Q$ now changes to problem $Q^{\prime}$ :

$$
\begin{array}{ll}
\left(Q^{\prime}\right) \quad \min & \sum_{j \in J} \varphi_{j}\left(\hat{S}_{j}\right) \\
& \min \quad \sum_{j \in J} C_{j}\left(\hat{S}_{j}\right) \\
& \text { subject to } \hat{S}_{j} \in \Phi_{j}^{\prime} \quad \forall j \in J .
\end{array}
$$

For problem $Q^{\prime}$, we are now able to construct a set of efficient solutions by a greedy algorithm (see e.g. Fox $(1966)$ ), because $C_{j}\left(\hat{S}_{j}\right)$ in increasing and convex on $\Phi^{\prime}$ and $\varphi_{j}\left(\hat{S}_{j}\right)$ is decreasing and convex on $\Phi^{\prime}$. A first efficient solution can be computed by setting $\hat{S}_{j}=\left\lceil\hat{\lambda}_{j} \hat{t}_{j}-1\right\rceil, \forall j \in J$. This solution is efficient (as long as $C(\hat{\mathbf{S}}) \leq C^{\text {obj}}$ ), because it has the lowest possible total SRU holding costs. Next, we can compute a 'greedy factor', representing the decrease in $\varphi_{j}\left(\hat{S}_{j}\right)$, equal to $-\Delta \varphi_{j}\left(\hat{S}_{j}\right)$, relative to the increase in $C_{j}\left(\hat{S}_{j}\right)$, which is equal to $\Delta C_{j}\left(\hat{S}_{j}\right)$. The 'greedy factor' $\tau_{j}$ for each $\operatorname{SRU} j \in J$ is equal to:

$$
\tau_{j}=\frac{\hat{\lambda}_{j} P\left(X_{j}=\hat{S}_{j}\right)}{\hat{h}_{j} \sum_{x=0}^{\hat{S}_{j}} P\left(X_{j}=x\right)}, \quad \hat{S}_{j} \geq \hat{\lambda}_{j} \hat{t}_{j}-1, \quad j \in J
$$

The base stock level of the SRU $j^{*} \in J$ with highest value of $\tau_{j}$ is increased by one unit and a new 'greedy factor' $\tau_{j^{*}}$ is determined based on the new base stock level $\hat{S}_{j^{*}}$. This procedure is repeated

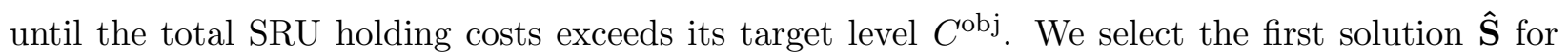

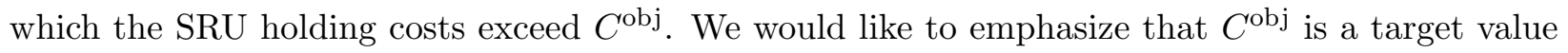


and should not be interpreted as a constraint, and for this reason we need not necessarily take the second to last solution.

\section{$5 \quad$ Capacity priority policies}

The second input for our simulation model is the choice of the capacity priority policy. Note that, because of our model assumptions, we are restricted to policies that do not take into account information on the actual ready-for-use LRU stock. We construct two 'extreme' policies (an inspection and a repair priority policy) in which inspection (repair) has priority, and multiple hybrid priority policies that, based on whether the repair workload in the shop is below or above a certain threshold, give priority to either inspection or repair of parts. In this section we motivate which policies we compare and we explain how each policy gives priority to either inspection or repair of parts.

Giving priority to inspection of failed parts facilitates earlier sourcing of the required SRUs and it potentially reduces the probability that the server becomes idle while there are still parts in the repair shop (but waiting for SRUs), which could reduce the actual server utilization. On the other

hand, it delays the repair and hence the availability of ready-for-use LRUs. In addition, separating inspection and repair may increase the repair time and is hence inefficient from a server utilization point of view. Giving priority to the repair of parts has the opposite effect.

The effects seem to depend on the state of the system. Let us therefore first discuss whether and when inspection or repair should have priority. It is easy to see that situations in which the number of parts in the repair shop is high (either for an individual LRU or for all LRUs), coincide with situations in which the number of LRU backorders is high and/or the ready-for-use LRU stock is low. This is due to the fact the parts circulate in a closed-loop. Hence, we would ideally allocate capacity to repair when the number of parts in repair (or better: the corresponding workload) is high, in order to minimize the (probability of) backorders and the corresponding costs. When the number of parts in repair is low, the probability of backorders will also be low and hence in this situation, giving priority to inspection could be beneficial.

For this reason, we will compare (repair) workload-based capacity priority policies. The service job workload, i.e. the time required to complete the repair phase of a service job, is (assumed to be) known after the inspection phase. Let $L$ be the repair workload of all service jobs that are 
either in repair or "ready-to-repair", and let $\breve{L}(\geq 0)$ be the workload threshold, based on which will be determined whether priority should be given to inspection or to repair. Note that if either the queue of jobs waiting for inspection or repair is empty, then always priority is given to the first job in the non-empty queue. Otherwise, if $L<\breve{L}$, then priority is given to inspection and if $L \geq \breve{L}$, then priority is given to repair. By varying the values of $\breve{L}$, we can construct a series of capacity priority policies.

Note that we have two extreme policies. In case $\breve{L}=0$, then priority is always given to repair (provided that "ready-to-repair" parts are available). This policy we will refer to as 'repair priority policy' in the remainder of this paper. In case $\breve{L}=\infty$, then priority is always given to inspection (provided that parts that need inspection are available). This policy we will refer to as 'inspection priority policy' in the remainder of this paper. Any hybrid policy, i.e. in which $0<\breve{L}<\infty$, will be referred to as 'hybrid priority policy'.

\section{Simulation}

Given a predetermined vector of SRU base stock levels $\hat{\mathbf{S}}$ and a choice of the capacity priority policy $\pi$, we are able to simulate the system (performance). The outcome of the simulation are

the variables $A_{i}(\hat{\mathbf{S}}, \pi, q)$ and $B_{j}(\hat{\mathbf{S}}, \pi, k)$, which are used to determine the total system costs. The performance of the system is evaluated via discrete event simulation. We use the same random seed in each simulation run for a fair comparison of the system performance under different choices of the SRU base stock levels and capacity priority policy. This means that we repeatedly use the same stream of random failures of LRUs, random inspection and repair times and random sets of SRUs required to repair failed parts.

We use the method of non-overlapping batch means (NOBM) to construct a $(1-\epsilon) \%$-confidence interval around the steady-state mean total costs; see Steiger and Wilson (2001) for a description of this method. In setting up the simulation experiments, we follow the guidelines in Schmeiser (1982) regarding the batch size and length of the simulation run, in order to avoid simulation biases and to make sure that the batch means are approximately i.i.d. normal variables. We divide the sequence of simulated outputs into a warm-up period of 100,000 demands and 20 batches of initial size 100,000 demands. 
We determine the steady-state distribution of the (assigned and unassigned) on-hand stock $B_{j}(\hat{\mathbf{S}}, \pi, k)$ of SRU $j, \forall j \in J$, for each batch separately. The steady-state distribution $A_{i}(\hat{\mathbf{S}}, \pi, q)$ of the number of parts of LRU $i$ in the repair shop is determined over all 20 batches together. Based on the steady state distributions $A_{i}(\hat{\mathbf{S}}, \pi, q)$ we are able to determine an optimal vector of LRU base stock levels $\mathbf{S}$. With equation (1), we are able to determine the simulated total costs for each batch separately. The batch mean and standard deviation of the total costs is determined and based on this, a $(1-\epsilon) \%$-confidence interval around the steady-state mean total costs is constructed.

The length of the simulation run depends on the desired accuracy of the found solution, and is chosen such that the probability that the simulated total costs are within $1 \%$ of its expected value is at least $95 \%$. As long as the desired accuracy is not reached, we double the batch size and combine available batches into new batch results. To avoid long run times, the simulation is always terminated when the batch size has reached 400,000. This stopping criterion was however not needed in the numerical study presented in Section 7.

\section{Numerical study}

In this section, we present the results of our simulation studies for a single representative setting, under the approaches for setting the SRU base stock levels as described in Section 4. In Section 7.1 we describe the setting, which represents a real-life repair shop environment with high material uncertainty, based on quantitative and qualitative data that are collected during interviews and plant visits in case studies at three companies in The Netherlands (see Chapter 5 in Driessen (2018)). In Section 7.2 we present the results of our simulation studies for this setting.

\section{$7.1 \quad$ Setting}

We consider a setting in which a single repair man is responsible for the repair of 20 LRUs. The LRUs vary in demand rate (5 levels) and price (4 levels) and each combination occurs once, see Table 1 for an overview. We have numbered the LRUs A1, .., A4, .., E1, ..., E4 for later references. The letter relates to the demand class $(\mathrm{A}=$ very fast moving, $\mathrm{E}=$ very slow moving) and the number relates to the price class ( $1=$ cheap item, $4=$ expensive item). The utilization of the repair man is at most $90 \%$, which is the case if all service jobs are interrupted after inspection. 
Table 1: LRU characteristics

\begin{tabular}{lcccccc}
\hline $\begin{array}{l}\text { Demand } \\
\text { class }\end{array}$ & $\begin{array}{c}\text { Demand rate } \\
\text { per time unit }\end{array}$ & $\begin{array}{c}\text { Price } \\
40.000\end{array}$ & 160.000 & 280.000 & 400.000 & $\begin{array}{c}\text { Inefficiency } \\
\text { factor (\%) }\end{array}$ \\
\hline Very fast moving & 0.1126 & A1 & A2 & A3 & A4 & $15 \%$ \\
Fast moving & 0.0563 & B1 & B2 & B3 & B4 & $20 \%$ \\
Medium moving & 0.0282 & C1 & C2 & C3 & C4 & $25 \%$ \\
Slow moving & 0.0141 & D1 & D2 & D3 & D4 & $30 \%$ \\
Very slow moving & 0.0093 & E1 & E2 & E3 & E4 & $50 \%$ \\
\hline
\end{tabular}

The total service job workload (inspection plus repair time) of each LRU is Gamma distributed, with an average of one time unit and standard deviation 0.5. The inspection time of a service job always equals $10 \%$ of the simulated total service job workload. The repair time equals $90 \%$ of the simulated total workload.

For service jobs that are interrupted for more than $T^{M a x}$ units after inspection, the repair time is increased with the inefficiency factor multiplied by the inspection time of that specific service job. In general, the 'inefficiency' is higher for parts that are repaired less often, because the part is more unknown to the repair man. The inefficiency factors hence increase when LRU demand rates decrease and can be found in Table 1; the average inefficiency factor (weighted for the various demand rates) is equal to $20 \%$.

Each LRU contains 50 SRUs and each SRU has a probability of $20 \%$ that it is required during a service job (i.e. $\alpha_{i, j}=0.2 \forall i \in I$ and $\forall j \in J$ ). We assume that each SRU is required for at most one LRU (no commonality), hence we have $50 \cdot 20=1000$ SRUs. The prices of the SRUs are a fixed percentage of the LRU price for which the SRUs are required. Note that the joint values of all SRUs do not necessarily represent the same value as the LRU to which they belong, but in fact can have any value. Table 2 provides an overview of the SRU classes we have, including the number of SRUs in that class and their price (related to the price of the LRU). Each SRU in a certain class has the same characteristics.

All SRUs are consumable and have a deterministic replenishment lead time, varying from 5 to 100 time units. The replenishment lead times are higher for SRUs with a higher price and/or lower demand rate. The weighted average lead time over all SRUs equals around 30 time units. The replenishment lead times range from 5 to 65 time units for SRUs related to LRU A1 and range from 40 to 100 time units for SRUs related to LRU E4. See table 3 for a complete overview of all SRU replenishment lead times. 
Table 2: SRU class characteristics for each LRU

\begin{tabular}{ccc}
\hline $\begin{array}{c}\text { SRU } \\
\text { class }\end{array}$ & $\begin{array}{c}\text { Number } \\
\text { of SRUs }\end{array}$ & $\begin{array}{c}\text { Price (\% of } \\
\text { LRU price) }\end{array}$ \\
\hline 1 & 10 & $0.25 \%$ \\
2 & 10 & $0.5 \%$ \\
3 & 10 & $1.0 \%$ \\
4 & 10 & $1.5 \%$ \\
5 & 5 & $2.0 \%$ \\
6 & 1 & $5.0 \%$ \\
7 & 1 & $10.0 \%$ \\
8 & 1 & $15 \%$ \\
9 & 1 & $20 \%$ \\
10 & 1 & $25 \%$ \\
\hline
\end{tabular}

Table 3: SRU replenishment lead times per SRU class per LRU (in time units)

\begin{tabular}{ccccccccccc}
\hline & \multicolumn{1}{c}{ SRU class } \\
LRU & 1 & 2 & 3 & 4 & 5 & 6 & 7 & 8 & 9 & 10 \\
\hline A1 & 5 & 10 & 15 & 20 & 25 & 30 & 35 & 45 & 55 & 65 \\
A2 & 10 & 15 & 20 & 25 & 30 & 35 & 40 & 50 & 60 & 70 \\
A3 & 15 & 20 & 25 & 30 & 35 & 40 & 45 & 55 & 65 & 75 \\
A4 & 20 & 25 & 30 & 35 & 40 & 45 & 50 & 60 & 70 & 80 \\
B1 & 10 & 15 & 20 & 25 & 30 & 35 & 40 & 50 & 60 & 70 \\
B2 & 15 & 20 & 25 & 30 & 35 & 40 & 45 & 55 & 65 & 75 \\
B3 & 20 & 25 & 30 & 35 & 40 & 45 & 50 & 60 & 70 & 80 \\
B4 & 25 & 30 & 35 & 40 & 45 & 50 & 55 & 65 & 75 & 85 \\
C1 & 15 & 20 & 25 & 30 & 35 & 40 & 45 & 55 & 65 & 75 \\
C2 & 20 & 25 & 30 & 35 & 40 & 45 & 50 & 60 & 70 & 80 \\
C3 & 25 & 30 & 35 & 40 & 45 & 50 & 55 & 65 & 75 & 85 \\
C4 & 30 & 35 & 40 & 45 & 50 & 55 & 60 & 70 & 80 & 90 \\
D1 & 20 & 25 & 30 & 35 & 40 & 45 & 50 & 60 & 70 & 80 \\
D2 & 25 & 30 & 35 & 40 & 45 & 50 & 55 & 65 & 75 & 85 \\
D3 & 30 & 35 & 40 & 45 & 50 & 55 & 60 & 70 & 80 & 90 \\
D4 & 35 & 40 & 45 & 50 & 55 & 60 & 65 & 75 & 85 & 95 \\
E1 & 25 & 30 & 35 & 40 & 45 & 50 & 55 & 65 & 75 & 85 \\
E2 & 30 & 35 & 40 & 45 & 50 & 55 & 60 & 70 & 80 & 90 \\
E3 & 35 & 40 & 45 & 50 & 55 & 60 & 65 & 75 & 85 & 95 \\
E4 & 40 & 45 & 50 & 55 & 60 & 65 & 70 & 80 & 90 & 100 \\
\hline
\end{tabular}

The holding costs $h_{i}$ and $\hat{h}_{j}$ are equal to $0.1 \%$ of the part price per time unit. This corresponds to an annual holding costs rate of $12.5 \%$ (assuming 125 time units is equivalent with one year). This number is based on an average service time of 2 working days per service job and 250 working days a year, see Chapter 5 in Driessen (2018). The backorder costs $b$ are equal to 5,500 per time unit for each LRU. The cost factors are chosen such that the average value of $\left(b-h_{i}\right) / b$ equals 0.96 over all LRUs $i \in I$. 


\subsection{Scenario results}

In this section, we present the results we have obtained under the different approaches for setting the SRU base stock levels. In Section 7.2.1 we present the results for the approach where all SRUs have an identical fill rate target $\gamma$, for $\gamma=\{0 \%, 50 \%, 75 \%, 90 \%, 95 \%\}$. The values for $\gamma$ relate to scenarios (0) up to (4) respectively, and correspond to low (scenarios (0) and (1)), medium (scenario (2)) and high (scenarios (3) and (4)) investments in SRUs. We make this distinction, because we observe that companies make different choices for investments in SRUs (low vs. high). The holding costs over the unassigned on-hand SRU stock (see equation (2)) based on the scenarios, in which all SRUs have an identical fill rate target, serve as a target $\left(C^{o b j}\right)$ for the holding costs over the unassigned on-hand SRU stock under the use of approach 2 for the SRUs. In this way, we get a fair comparison between the approaches 1 and 2 for the SRUs under each of the scenarios. The values of $\gamma$ and $C^{o b j}$ for each of the scenarios are listed in Table 4 (the listed values were obtained after applying approach 1; see Table 6).

Table 4: Definition of $\gamma$ (used by approach 1 for the SRUs) and $C^{o b j}$ (used by approach 2 for the SRUs) for the scenarios (0)-(4).

\begin{tabular}{ccc}
\hline Scenario & $\gamma$ & $C^{\text {obj }}$ \\
\hline$(0)$ & $0 \%$ & 0.00 \\
$(1)$ & $50 \%$ & $4,408.69$ \\
$(2)$ & $75 \%$ & $6,131.27$ \\
$(3)$ & $90 \%$ & $9,690.07$ \\
$(4)$ & $95 \%$ & $11,571.96$ \\
\hline
\end{tabular}

We study two options: the 'inefficiency' either is obtained directly (see Sections 7.2.1 and 7.2.2), i.e. $T^{M a x}=0$, or only after a certain period (Section 7.2 .3 ), in this specific case $T^{\text {Max }}$ equals 5.0 time units. In the latter case the 'inefficiency' is absent, if the repair phase starts within 5.0 time units after the inspection phase was completed. Section 7.2.4 concludes this section with the insights from the numerical study.

For each scenario, we present the total system costs per time unit for various values of the workload threshold $\breve{L}$. From this, one can determine the optimal workload threshold value for each scenario. Further, we present more detailed results for each scenario under the optimal workload threshold value. The 'Total SRU holding costs' here represent the average holding costs per time calculated over the assigned and unassigned on-hand SRU stock. Most of the other characteristics 
are intuitively clear, some of them are explained hereafter:

1. Time repair shop empty: percentage of time that the repair shop is empty.

2. Average waiting time SRUs job complete: average time from completion of the inspection phase of a random service job until the moment that all required SRUs are available from stock.

3. Average time waiting capacity: average time that a random service job is waiting for capacity (in the buffer "Failed LRUs" or in the buffer "Ready-to-repair")

4. Average time in service: average time that a random service job is in service (inspection plus repair) by the repair man.

\subsubsection{Scenario results if all SRUs have an identical fill rate target $\gamma$}

Table 5 shows the total system costs for the approach in which all SRUs have an identical fill rate target, for different combinations of scenarios and capacity priority policies (i.e. workload thresholds $\breve{L}$ ). For scenarios with low or medium SRU investments (scenarios (0), (1) and (2)), an inspection $(\breve{L}=\infty)$ or hybrid priority policy is the best choice. For scenarios with high SRU investments (scenarios (3) and (4)) the repair priority policy is the best choice. An individual fill rate target of $90 \%$ (scenario (3)) in combination with a repair priority policy leads to the lowest total system costs of 21,058.79 per time unit; the difference in total system costs under this scenario but with different capacity priority policies is at most $4.15 \%$.

Table 6 shows the detailed results for the same scenarios under the optimal value of the workload threshold, i.e. the workload threshold that minimizes the total system costs. From this table, we see that increasing the investments in SRUs positively influences the service job lead times in two ways. Firstly, the average waiting time until all SRUs for a random job are 'complete', decreases. Secondly, the number of inefficient repairs decreases (especially under the repair priority policy), which results in a lower server utilization and a lower waiting time for capacity. 
Table 5: Total costs per time unit if all SRUs have an identical fill rate target $\gamma$

\begin{tabular}{cccccc}
\hline$\breve{L}$ & $(0)$ & $(1)$ & $(2)$ & $(3)$ & $(4)$ \\
\hline 0.0 & $55,742.89$ & $26,318.37$ & $22,433.93$ & $\mathbf{2 1 , 0 5 8 . 7 9}$ & $\mathbf{2 1 , 2 8 8 . 1 0}$ \\
1.0 & $54,965.74$ & $26,108.42$ & $22,476.21$ & $21,228.97$ & $21,433.41$ \\
2.0 & $53,112.54$ & $25,257.55$ & $22,285.28$ & $21,526.94$ & $21,876.41$ \\
3.0 & $51,205.35$ & $24,581.50$ & $22,100.51$ & $21,570.12$ & $22,017.17$ \\
4.0 & $49,119.39$ & $24,106.39$ & $21,986.73$ & $21,582.43$ & $22,060.77$ \\
5.0 & $46,456.32$ & $23,813.60$ & $21,923.57$ & $21,601.22$ & $22,100.80$ \\
6.0 & $43,091.67$ & $23,621.02$ & $21,891.22$ & $21,623.53$ & $22,137.44$ \\
7.0 & $39,254.73$ & $23,515.44$ & $21,882.95$ & $21,652.68$ & $22,174.80$ \\
8.0 & $35,845.16$ & $23,449.86$ & $\mathbf{2 1 , 8 8 0 . 8 6}$ & $21,682.65$ & $22,212.37$ \\
9.0 & $33,450.33$ & $23,424.54$ & $21,893.58$ & $21,710.10$ & $22,244.59$ \\
10.0 & $31,760.20$ & $\mathbf{2 3 , 4 0 8 . 1 4}$ & $21,902.55$ & $21,734.63$ & $22,275.90$ \\
11.0 & $30,827.27$ & $23,413.57$ & $21,916.15$ & $21,757.75$ & $22,303.67$ \\
12.0 & $30,357.40$ & $23,414.19$ & $21,928.40$ & $21,780.53$ & $22,328.90$ \\
$\infty$ & $\mathbf{2 9 , 6 6 1 . 3 1}$ & $23,475.62$ & $22,011.89$ & $21,931.75$ & $22,493.75$ \\
\hline
\end{tabular}

Table 6: Detailed results for optimal capacity priority policy per scenario

\begin{tabular}{lccccc}
\hline Results per scenario & $(0)$ & $(1)$ & $(2)$ & $(3)$ & $(4)$ \\
\hline Optimal workload threshold & $\infty$ & 10.0 & 8.0 & 0.0 & 0.0 \\
Cost deviation w.r.t. repair priority policy & $87.93 \%$ & $12.43 \%$ & $2.53 \%$ & $0.00 \%$ & $0.00 \%$ \\
Cost deviation w.r.t. inspection priority policy & $0.00 \%$ & $0.29 \%$ & $0.60 \%$ & $4.15 \%$ & $5.66 \%$ \\
Total costs per time unit & $29,661.31$ & $23,408.14$ & $21,880.86$ & $21,058.79$ & $21,288.10$ \\
Total LRU holding costs & $24,320.00$ & $14,080.00$ & $11,280.00$ & $8,040.00$ & $6,960.00$ \\
Total backorder costs & $4,589.84$ & $4,109.86$ & $3,918.39$ & $3,175.78$ & $2,678.61$ \\
Total SRU holding costs & 751.47 & $5,218.27$ & $6,682.47$ & $9,843.02$ & $11,649.49$ \\
Total assigned SRU holding costs & 751.47 & 806.08 & 548.25 & 150.19 & 74.73 \\
Total unassigned SRU holding costs & 0.00 & $4,412.20$ & $6,134.23$ & $9,692.83$ & $11,574.76$ \\
Target unassigned SRU holding costs $\left(C^{o b j}\right)$ & 0.00 & $4,408.69$ & $6,131.27$ & $9,690.07$ & $11,571.96$ \\
Time repair shop empty & $0.0000 \%$ & $0.0000 \%$ & $0.0313 \%$ & $1.1287 \%$ & $3.7290 \%$ \\
Aggregate SRU fill rate & $0.00 \%$ & $73.22 \%$ & $87.33 \%$ & $95.96 \%$ & $97.82 \%$ \\
SRU Job completeness & $0.00 \%$ & $22.93 \%$ & $44.30 \%$ & $74.85 \%$ & $84.63 \%$ \\
Percentage repair directly after inspection & $0.00 \%$ & $5.08 \%$ & $11.29 \%$ & $74.85 \%$ & $84.63 \%$ \\
Average waiting time SRUs job complete & 53.041 & 17.069 & 9.062 & 3.066 & 1.483 \\
Average time waiting capacity & 6.008 & 5.676 & 5.605 & 5.342 & 4.984 \\
Average time in service & 1.020 & 1.019 & 1.018 & 1.005 & 1.003 \\
Average service job lead time & 60.068 & 23.763 & 15.685 & 9.413 & 7.470 \\
\hline
\end{tabular}




\subsubsection{Scenario results if SRU job completeness is maximized}

Table 7 shows the total system costs per time unit for the approach in which we optimize the SRU job completeness, given a constraint on the holding costs over the unassigned on-hand SRU stock $\left(C^{o b j}\right.$, see Table 4$)$, for different combinations of scenarios and capacity priority policies (i.e. workload thresholds $\breve{L}$ ).

Table 7: Total costs per time unit if SRU job completeness is maximized

\begin{tabular}{cccccc}
\hline$\breve{L}$ & $(0)$ & $(1)$ & $(2)$ & $(3)$ & $(4)$ \\
\hline 0.0 & $55,742.89$ & $\mathbf{2 4 , 5 7 5 . 6 8}$ & $\mathbf{2 1 , 9 3 1 . 8 9}$ & $\mathbf{2 0 , 3 2 8 . 7 6}$ & $\mathbf{2 0 , 8 3 5 . 4 2}$ \\
1.0 & $54,965.74$ & $24,711.71$ & $22,061.67$ & $20,396.63$ & $20,877.44$ \\
2.0 & $53,112.54$ & $24,842.96$ & $22,380.23$ & $20,813.10$ & $21,228.35$ \\
3.0 & $51,205.35$ & $24,815.21$ & $22,431.84$ & $21,032.41$ & $21,520.89$ \\
4.0 & $49,119.39$ & $24,789.18$ & $22,444.73$ & $21,083.36$ & $21,576.43$ \\
5.0 & $46,456.32$ & $24,771.91$ & $22,460.92$ & $21,121.93$ & $21,614.80$ \\
6.0 & $43,091.67$ & $24,764.54$ & $22,476.26$ & $21,159.05$ & $21,651.06$ \\
7.0 & $39,254.73$ & $24,759.11$ & $22,491.79$ & $21,196.12$ & $21,684.30$ \\
8.0 & $35,845.16$ & $24,756.85$ & $22,510.54$ & $21,228.39$ & $21,711.72$ \\
9.0 & $33,450.33$ & $24,758.97$ & $22,528.20$ & $21,256.60$ & $21,739.12$ \\
10.0 & $31,760.20$ & $24,765.53$ & $22,545.14$ & $21,284.35$ & $21,765.85$ \\
11.0 & $30,827.27$ & $24,774.55$ & $22,561.02$ & $21,309.02$ & $21,789.00$ \\
12.0 & $30,357.40$ & $24,782.96$ & $22,575.59$ & $21,332.24$ & $21,810.94$ \\
$\infty$ & $\mathbf{2 9 , 6 6 1 . 3 1}$ & $24,874.56$ & $22,699.29$ & $21,503.51$ & $21,977.17$ \\
\hline
\end{tabular}

In all scenarios except scenario (0), we see that the repair priority policy is the best choice. This is in line with our findings in Section 7.2.1, in which we saw that the repair priority policy is the best choice if aggregate SRU fill rates are high. In the scenarios in which we optimize the SRU job completeness, the aggregate fill rates range from $94.91 \%$ to $99.63 \%$ (see Table 8 ).

By maximizing the SRU job completeness, we see that the total system costs decrease for scenarios (3) and (4): from 21,058.79 to 20,328.76 and from 21,288.10 to 20,835.42, respectively. From the evaluated scenarios we see that scenario (3), in which the SRU job completeness is maximized and a repair priority policy is applied, has the lowest total system costs.

In scenarios (1) and (2), we observe that the total system costs increase when we maximize the SRU job completeness. This can be explained by two factors. First, we see that different capacity priority policies (i.e. with a different optimal workload threshold) are the best choice in these scenarios. For example, in scenario (2) the total costs for approach 1 (equal parts fill rate) occurs for a threshold equal to 8.0 (smaller than the optimal system costs in scenario (2) for approach 2), while under a strict repair priority policy $(\breve{L}=0.0)$ the total system costs decrease from $22,433.93$ 
Table 8: Detailed results for optimal capacity priority policy per scenario

\begin{tabular}{lccccc}
\hline Results per scenario & $(0)$ & $(1)$ & $(2)$ & $(3)$ & $(4)$ \\
\hline Optimal workload threshold & $\infty$ & 0.0 & 0.0 & 0.0 & 0.0 \\
Cost deviation w.r.t. repair priority policy & $87.93 \%$ & $0.00 \%$ & $0.00 \%$ & $0.00 \%$ & $0.00 \%$ \\
Cost deviation w.r.t. inspection priority policy & $0.00 \%$ & $1.22 \%$ & $3.50 \%$ & $5.78 \%$ & $5.48 \%$ \\
Total costs per time unit & $29,661.31$ & $24,575.68$ & $21,931.89$ & $20,328.76$ & $20,835.42$ \\
Total LRU holding costs & $24,320.00$ & $16,440.00$ & $11,760.00$ & $8,000.00$ & $6,400.00$ \\
Total backorder costs & $4,589.84$ & $3,166.62$ & $3,679.68$ & $2,485.13$ & $2,817.19$ \\
Total SRU holding costs & 751.47 & $4,969.06$ & $6,492.21$ & $9,843.63$ & $11,618.23$ \\
Total assigned SRU holding costs & 751.47 & 557.38 & 324.60 & 94.67 & 39.25 \\
Total unassigned SRU holding costs & 0.00 & $4,411.68$ & $6,167.61$ & $9,748.96$ & $11,578.98$ \\
Target unassigned SRU holding costs $\left(C^{\text {obj }}\right)$ & 0.00 & $4,408.69$ & $6,131.27$ & $9,690.07$ & $11,571.96$ \\
Time repair shop empty & $0.0000 \%$ & $0.0000 \%$ & $0.0149 \%$ & $2.7776 \%$ & $6.2960 \%$ \\
Aggregate SRU fill rate & $0.00 \%$ & $94.91 \%$ & $97.29 \%$ & $99.21 \%$ & $99.63 \%$ \\
SRU Job completeness & $0.00 \%$ & $66.52 \%$ & $80.28 \%$ & $93.32 \%$ & $96.75 \%$ \\
Percentage repair directly after inspection & $0.00 \%$ & $66.52 \%$ & $80.28 \%$ & $93.32 \%$ & $96.75 \%$ \\
Average waiting time SRUs job complete & 53.041 & 15.414 & 8.385 & 1.824 & 0.781 \\
Average time waiting capacity & 6.008 & 6.230 & 5.417 & 4.847 & 4.751 \\
Average time in service & 1.020 & 1.007 & 1.005 & 1.002 & 1.001 \\
Average service job lead time & 60.068 & 22.652 & 14.807 & 7.673 & 6.533 \\
\hline
\end{tabular}

(scenario (1)) to 21,931.89 (scenario (2)).

Second, in scenario (1) and (2) under maximization of the SRU job completeness, we see that especially expensive SRUs, that are (potentially) required for the repair of expensive, slow moving LRUs (D4, E3 and E4) are not being stocked. This causes high waiting times for these specific LRUs and consequently higher (holding plus backordering) costs for LRUs. We don't see this phenomenon in scenarios (3) and (4). In these scenarios all SRU base stock levels are at least equal to 1 and, because the largest part of the individual SRU waiting times is decreased by increasing the base stock level from 0 to 1, extremely long repair lead times for expensive slow moving LRUs (like D4, E3 and E4) almost never occur. This significantly reduces the LRU costs.

Inspection and hybrid priority policies tend to work well if many SRU base stock levels are equal to zero. We observe this phenomenon in scenarios (1) and (2), in which the total costs increased when the SRU job completeness is maximized (and the number of SRUs with base stock level 0 increased). Service jobs that require SRUs with base stock level 0 directly benefit from earlier ordering of these SRUs. Demands for SRUs with base stock level greater than 0 are fulfilled from stock or by replenishment orders that are generated by earlier demands. Hence, a service job that only requires SRUs with base stock levels greater than 0, benefits less strongly from an early inspection. 
Table 9: Total costs per time unit if inefficiency only occurs after 5 time units and SRU job completeness is maximized

\begin{tabular}{cccccc}
\hline$\breve{L}$ & $(0)$ & $(1)$ & $(2)$ & $(3)$ & $(4)$ \\
\hline 0 & $55,742.89$ & $24,563.30$ & $\mathbf{2 1 , 9 2 1 . 6 7}$ & $\mathbf{2 0 , 3 2 1 . 0 8}$ & $\mathbf{2 0 , 8 3 0 . 5 1}$ \\
1 & $54,965.74$ & $24,554.74$ & $21,924.34$ & $20,326.97$ & $20,833.96$ \\
2 & $53,112.54$ & $24,495.84$ & $21,929.91$ & $20,377.35$ & $20,880.48$ \\
3 & $51,205.35$ & $24,451.57$ & $21,935.75$ & $20,430.08$ & $20,943.26$ \\
4 & $49,119.39$ & $\mathbf{2 4 , 4 3 5 . 0 7}$ & $21,956.14$ & $20,474.84$ & $20,988.37$ \\
5 & $46,456.32$ & $24,574.50$ & $22,186.48$ & $20,792.40$ & $21,303.05$ \\
6 & $43,091.67$ & $24,676.95$ & $22,370.09$ & $21,055.97$ & $21,567.51$ \\
7 & $39,254.73$ & $24,678.39$ & $22,394.42$ & $21,106.68$ & $21,611.83$ \\
8 & $35,845.16$ & $24,678.73$ & $22,413.99$ & $21,139.17$ & $21,642.30$ \\
9 & $33,450.33$ & $24,681.44$ & $22,434.87$ & $21,167.06$ & $21,669.65$ \\
10 & $31,760.20$ & $24,686.84$ & $22,450.18$ & $21,194.70$ & $21,696.19$ \\
11 & $30,827.27$ & $24,696.68$ & $22,466.48$ & $21,219.62$ & $21,719.51$ \\
12 & $30,357.40$ & $24,705.81$ & $22,483.23$ & $21,242.57$ & $21,740.81$ \\
$\infty$ & $\mathbf{2 9 , 6 6 1 . 3 1}$ & $24,796.75$ & $22,607.43$ & $21,407.46$ & $21,902.17$ \\
\hline
\end{tabular}

\subsubsection{Scenario results if inefficiency only occurs after 5 time units}

Table 9 shows the total system costs for the approach in which we optimize the SRU job completeness, given a constraint on the holding costs over the unassigned on-hand SRU stock, for different combinations of scenarios and capacity priority policies when the inefficiency is only obtained after $T^{M a x}=5.0$ time units. For scenario (1), we now see that a hybrid priority policy (with $\breve{L}=4.0$ ) is the best choice. In this situation, the total system costs are $0.5 \%$ lower than in the repair priority policy. However, we still see that, under the evaluated scenarios, scenario (3) in combination with a repair priority policy leads to the lowest total system costs.

\subsubsection{Insights from numerical study}

In this section, we have evaluated workload-based capacity priority policies in a representative setting for several scenarios, corresponding to low (scenarios (0) and (1)), medium (scenario (2)) and high (scenarios (3) and (4)) investments in SRUs, and approaches to set the SRU base stock levels. The combinations of the approach for the SRUs and capacity priority policy that lead to the lowest total costs per scenario are listed in Table 10.

From the evaluation study, we can draw the following conclusions:

1. In a setting with low and medium investments in SRUs (scenarios (0), (1) and (2)), inspection and hybrid priority policies in combination with a simple approach (1) to set the SRU base 
Table 10: Approach for the SRUs and capacity priority policy that lead to the lowest total costs per scenario.

\begin{tabular}{cccccc}
\hline Results per scenario & $(\mathbf{0})$ & $\mathbf{( 1 )}$ & $\mathbf{( 2 )}$ & $\mathbf{( 3 )}$ & $\mathbf{( 4 )}$ \\
\hline Approach SRUs & 1 or 2 & 1 & 1 & 2 & 2 \\
Workload threshold $(\breve{L})$ & $\infty$ & 10.0 & 8.0 & 0.0 & 0.0 \\
Capacity priority policy & Inspection & Hybrid & Hybrid & Repair & Repair \\
Total costs & $29,661.31$ & $23,408.14$ & $21,880.86$ & $\mathbf{2 0 , 3 2 8 . 7 6}$ & $20,835.42$ \\
\hline
\end{tabular}

stock levels, outperform repair priority policies (independent of the SRU approach). Due to the limited investment in SRUs, service jobs will often have to wait for missing SRUs. In such a setting, it is best to level out the waiting times across the SRUs (which is the case in the first SRU approach) and to prioritize inspections (to speed up sourcing SRUs).

2. In a setting with a high investment in SRUs (scenarios (3) and (4)), independent of the SRU approach, repair priority policies outperform inspection and hybrid priority policies. This also holds for situations in which the inefficiency appears only after a certain time period. The high investment in SRUs enable short waiting times for SRUs (all SRU base stock levels are at least 1), and a smooth repair process is enabled (i.e. low number of service job interruptions because of missing SRUs) by maximizing the SRU job completeness.

3. The lowest total costs are obtained under a high investment in SRUs (scenario (3)), the use of the SRU job completeness approach and the use of the repair priority policy.

Notice that these conclusions hold for our representative setting. Very different values for e.g. the ratio of inspection times over repair times, the inefficiency factor, the ratio of costs of SRUs vs. costs of LRUs, and the factors $\left(b-h_{i}\right) / b$, may lead to different conclusions.

\section{Conclusions}

In this paper, we have studied a repairable parts inventory system in which multiple, two-indentured LRUs are repaired by a repair shop consisting of a two-stage service process facing a high material uncertainty. The first stage is the inspection phase, that is needed to determine which SRUs are required in the second (repair) phase. Inspection and repair both take a significant amount of time, they are executed by a group of repair men, and repair times depend on the time that elapses 
between inspection and the repair of a part. The service time gets longer once service jobs are interrupted, because the inspected part needs to be put aside and the repair man needs to recall the problem essentials when the repair starts.

We model the repair capacity as a single repair man facing two queues: one of parts still to be inspected (and to determine what is needed to repair them) and one of parts already inspected for which materials needed are available in stock. Both queues are served on a first-come first-serve basis, the question is when to switch between the two queues. We have evaluated workload-based capacity priority policies in an 'extreme' but representative setting for several scenarios (i.e. low, medium and high investments in SRUs) and two approaches to set the SRU base stock levels. From the scenarios we have evaluated, a scenario with high investments in SRUs, in which the SRU job completeness is maximized and in which a repair priority policy is applied leads to the lowest total costs.

The evaluation is limited to a single setting, but we stipulate that the setting has been chosen on purpose and that results are generalizable. More specific, the setting is based on an 'extreme' situation where the repair shop faces a high material uncertainty, that is represented by low SRU failure rates and relatively high prices for the SRUs. Given the results it is clear that we do not have to present less 'extreme' situations, i.e. where the material uncertainty is lower, as the conclusions will be similar. Furthermore, we chose for the 'extreme case' of one server on purpose, because this setting forces one to choose between different priorities. We expect insights do not change if we would apply the same logic in a multi-server setting, but instead some effects might remain more hidden.

The results are interesting in three ways. First, we observe that different combinations of approaches to set the SRU base stock levels and capacity priority policies are optimal under different (low, medium, high) investments in SRUs. Second, we observe that a rather simple capacity priority rule is optimal under high investments in SRUs, which simplifies application. Third, the cost difference between a smart (approach 2) and rather simple method (approach 1) to set the SRU base stock levels, under high investments in SRUs, is significant (3.5\%). Yet, to come to these conclusions we made some simplifying assumptions. These assumptions possibly influence the optimal capacity priority policy, and future studies should investigate the impact of their relaxation. 
One extension could be to incorporate priority rules for executing service jobs (instead of the FCFS service discipline). Our model is, with some simple extensions, also applicable for static capacity priority policies. For example, (at least) two priority classes could be created, of which one class consists of the LRUs for which the expected SRU job completeness is relatively low. Note that the assignment to priority classes can be done upfront, as we also determine the SRU base stock levels upfront. In a new, to be defined capacity priority policy one could give priority to inspection of the parts that have a relatively high probability of being interrupted because of missing SRUs. Another extension could be the use of dynamic (or myopic) priority policies (i.e. policies that are based on the actual LRU stock levels), but note that this requires a different modeling approach.

\section{References}

I.J.B.F. Adan, A. Sleptchenko, and G.J. Van Houtum. Reducing costs of spare parts supply systems via static priorities. Asia-Pacific Journal of Operational Research, 26(4):559-585, 2009.

S.C. Albright and A. Gupta. Steady-state approximation of a multi-echelon, multi-indentured repairable-item inventory system with a single repair facility. Naval Research Logistics, 40:479-493, 1993.

J.J. Arts. A multi-item approach to repairable stocking and expediting in a fluctuating demand environment. European Journal of Operational Research, 256:102-115, 2017.

J.A. Buzacott. Queueing models of Kanban and MRP controlled production systems. Engineering Costs and Production Economics, 17:3-20, 1989.

J.A. Buzacott and J.G. Shanthikumar. Stochastic models of manufacturing systems, volume 4. Englewood Cliffs, NJ: Prentice Hall, 1993.

Y. Dallery and G. Liberopoulos. Extended Kanban control system: Combining Kanban and base stock. IIE Transactions, 32(4):369-386, 2000.

M.A. Driessen. Integrated Capacity Planning and Inventory Control for Repairable Spare Parts. PhD thesis, Eindhoven University of Technology, School of Industrial Engineering, 2018. Available at: research.tue.nl.

M.A. Driessen, J.W. Rustenburg, G.J. Van Houtum, and V.C.S. Wiers. Connecting inventory and repair shop control for repairable items. In H. Zijm, M. Klumpp, U. Clausen, and M. Hompel, editors, Logistics and Supply Chain Innovation. Lecture Notes in Logistics, pages 199-221. Springer, Cham, 2015.

B. Fox. Discrete optimization via marginal analysis. Management Science, 13(3):210-216, 1966.

Y. Frein, M. Di Mascolo, and Y. Dallery. On the design of Generalized Kanban control systems. International Journal of Operations $\&$ Production Management, 15(9):158-184, 1995.

A. Gupta and S.C. Albright. Steady-state approximations for a multi-echelon, multi-indentured repairable iteminventory system. European Journal of Operational Research, 62:340-353, 1992.

W.J. Hopp and M.L. Spearman. Factory physics. McGraw-Hill, 2001.

C. Kohler-Gudum and A.G. de Kok. A safety stock adjustment procedure to enable target service levels in simulation of generic inventory systems. Beta working paper 71, 2002.

E. Levner, Y. Perlman, T.C.E. Cheng, and I. Levner. A network approach to modeling the multi-echelon spare-part inventory system with backorders and interval-valued demand. International Journal of Production Economics, 132:43-51, 2011. 
J.A. Muckstadt. A model for a multi-item, multi-echelon, multi-indenture inventory system. Management Science, 20(4):472-481, 1973.

W.D. Rustenburg, G.J. van Houtum, and W.H.M. Zijm. Exact and approximate analysis of multi-echelon, multiindenture spare parts systems with commonality. In J.G. Shanthikumar, D.D. Yao, and W.H.M. Zijm, editors, Stochastic Modeling and Optimization of Manufacturing Systems and Supply Chains, pages 143-176. Kluwer Academic, Boston/Dordrecht/London, 2003.

B.W. Schmeiser. Batch size effects in the analysis of simulation output. Operations Research, 30(3):556-568, 1982.

C.C. Sherbrooke. METRIC: A multi-echelon technique for recoverable item control. Operations Research, 16(1): $122-141,1968$.

C.C. Sherbrooke. VARI-METRIC: Improved approximations for multi-indenture, multi-echelon availability models. Operations Research, 34(2):311-319, 1986.

C.C. Sherbrooke. Optimal inventory modeling of systems: Multi-echelon techniques. Wiley, 2 edition, 2004.

A. Sleptchenko, M.C. van der Heijden, and A. van Harten. Effects of finite repair capacity in multi-echelon, multiindenture service part supply systems. International Journal of Production Economics, 79:209-230, 2002.

A. Sleptchenko, M.C. van der Heijden, and A. van Harten. Trade-off between inventory and repair capacity in spare part networks. Journal of the Operational Research Society, 54(3):263-272, 2003.

A. Sleptchenko, M.C. van der Heijden, and A. van Harten. Using repair priorities to reduce stock investment in spare part networks. European Journal of Operational Research, 163:733-750, 2005.

N.M. Steiger and J.R. Wilson. Convergence properties of the batch means method for simulation output analysis. INFORMS Journal on Computing, 13(4):277-293, 2001.

O. Stenius, J. Marklund, and S. Axsäter. Sustainable multi-echelon inventory control with shipment consolidation and volume dependent freight costs. European Journal of Operational Research, 267:904-916, 2018.

H.G.H. Tiemessen. Dynamic allocation in multi-dimensional inventory models. PhD thesis, Eindhoven University of Technology, School of Industrial Engineering, 2014. Available at: research.tue.nl.

H.G.H. Tiemessen and G.J. van Houtum. Reducing costs of repairable inventory supply systems via dynamic scheduling. International Journal of Production Economics, 143(2):478-488, 2013.

G.J. Van Houtum and A.A. Kranenburg. Spare Parts Inventory Control under System Availability Constraints. New York: Springer, 1st edition, 2015.

W. Van Jaarsveld, T. Dollevoet, and R. Dekker. Improving spare parts inventory control at a repair shop. Omega, $57: 217-229,2015$.

W.H.M. Zijm and Z.M. Avşar. Capacitated two-indenture models for repairable item systems. International Journal of Production Economics, 81-82:573-588, 2003. 